\title{
Tinea corporis caused by Microsporum canis in HIV patient treated for neuromeningeal cryptococcis: report of a nosocomial outbreak
}

\author{
Merad Yassine ${ }^{1}$, Adjmi-Hamoudi Haiet ${ }^{2}$, Tabet-Derraz Narjess ${ }^{3}$, Merad Fatima Zohra Samia ${ }^{4}$
}

\begin{abstract}
We report a nosocomial outbreak of Microsporum canis during inpatient treatment for HIV newly diagnosed with cryptococcal meningitis. The clinical presentation, though very typical of ringworm infection, samples were collected and examined by direct microscopy and culture on Sabouraud's dextrose agar, revealing Microsporum canis. It is strongly believed that outdoor cats living in the hospital spaces are the main cause of disease transmission. Measures to limit zoophilic pathogens are necessary in immunosuppressed inpatient care unit.
\end{abstract}

\section{Keywords}

Cryptococcus sp; Meningitis; Microsporum canis; Nosocomial; tinea corporis

\section{Introduction:}

Dermatophytes are keratinophiles and keratinolytic fungi, Microsporum canis remains a wide spread zoophilic dermatophyte among Algerian children ${ }^{1}$.

Dermatophytes are ascomycetes with septate hyphae, most closely related to coccidioides immitis within the Onygenales $^{2}$, they are three genera of dermatophytes, Trichophyton, Microporum, and Epidermophyton, devided by morphology and physical attributes.

There have been no reliable reports of dermatophyte nosocomial outbreak in immunocompromised patient. Although it is not possible to confirm thoroughly the source of the infection, this is an unusual nosocomial outbreak, this result emphasizes the problem of pets in the hospitals and the possible transmission of zoophilic pathogens.

\section{Case and methods:}

A 26 year old female patient newly diagnosed with HIV, admitted for therapeutic management of cryptococcal meningitis in the infectious diseases department. Physical examination of patient revealed a temperature of $38,5^{\circ} \mathrm{C}$, the white blood cell (WBC) count of 6000 cells $/ \mathrm{mm}^{3}$, patient was treated with intravenous fluconazole.

At day 7 of hospitalization the patient presented rounded, circinate and scaly patches on left arm, with an advancing active border and central clearing Figure 1 , the patient has also reported severe itching.

Skin scrapings from annular lesion were collected on sterile Petri dish and were inoculated on the surface of SGA (cyclohexemide and chloramphenicol) then incubated at $27^{\circ} \mathrm{C}$, after five days, it grows white and cottony with a yellow perimeter and a bright yellow orange underside, revealing Microsporum canisFigure 2, it has microscopically multicelled spores known as macroconidies with rough thick walls Figure 3 , the direct examination with $\mathrm{KOH}$ aids in visualising hyphae on skin scraping and confirming the diagnosis of dermatophyte.

\section{Discussion:}

Dermatophytes are a group of pathogenic fungi that cause mostly superficial diseases, further it is more difficult to diagnose dermatophytosis in immunocompromised patients, as clinical presentation is often atypical ${ }^{3}$, in rare cases dermatophyte infection may be invasive, especially in immunocompromised patients ${ }^{4}$.

Microsporum canis species is the most common pathogen in skin infections, Infections occurs by contact with arthrospores, some spread from animal hosts (zoophilic organisms), as well as indirectly from fomites (combs, brushes, hats, upholstery, furniture, linens), direct inoculation through breaks in the skin occurs more often in persons with depressed cell-mediated immunity ${ }^{5}$.

\footnotetext{
1 Department of parasitology-mycology, CHU "Hassani Abdelkader", UDL, Sidi-Bel-Abbès, Algeria

2 bDepartment of parasitology-mycology, University of medicine, Algiers, Algeria

3 Department of infectious diseases, CHU "Hassani Abdelkader", UDL, Sidi-Bel-Abbès, Algeria

4 Department of occupational medicine health, $\mathrm{CHU}$ "Hassani Abdelkader", Sidi-Bel-Abb`es, Algeria
}

Corresponding author:

Merad Yassine, Department of parasitology-mycology, CHU "Hassani Abdelkader", UDL, Sidi-Bel-Abbès, Algeria 


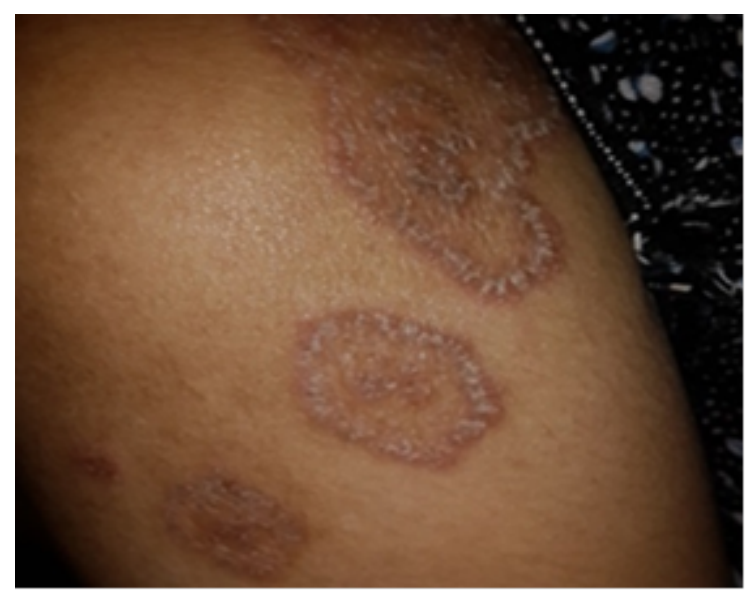

Figure 1. Tinea corporis in HIV inpatient at day 7 of hospitalisation

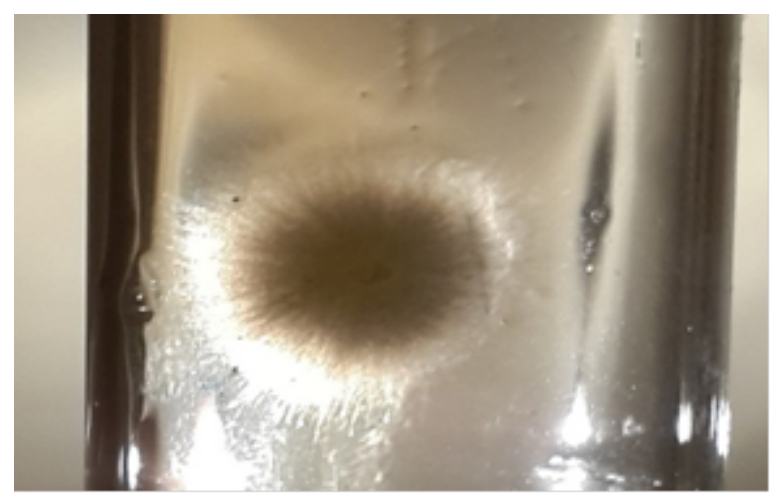

Figure 2. Microsporum canis at macroscopy

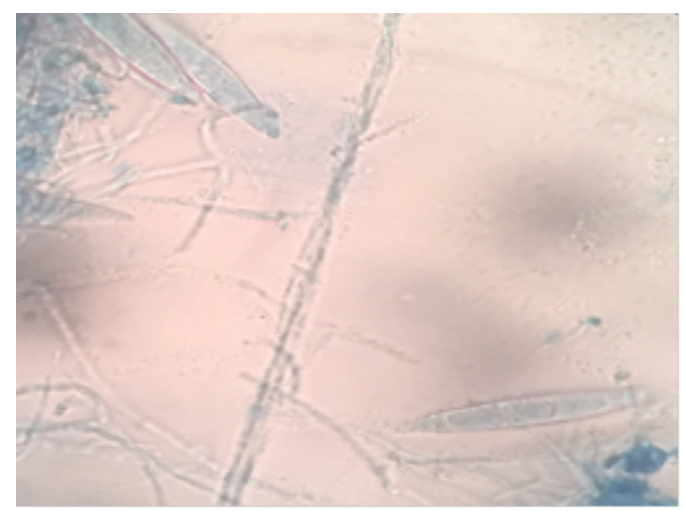

Figure 3. Microsporum canis at microscopy $\mathrm{X} 40$

Nosocomial infection by dermatophyte is already reported $^{6,7}$, a large outbreak of Trichosporon tonsurans was described among health care workers in a pediatric hospital ${ }^{8}$

Indirect transmission of tinea corporis caused by Microsporum canis was reported in hospital, after handling of contaminated laundry, after the use of shared razor ${ }^{9}$, by nurses working in a newborn unit to an infant ${ }^{10}$.

In our case the dermatophyte was apparently introduced into the facility by cats living in the garden of the hospital, the natural reservoir of Microsporum canis is in cats and dogs, nosocomial transmission of ringworm by cats was describe in neonatal intensive care unit ${ }^{11}$.
The unusual features of this clinical dermatophytosis were the intrahospital localization of this zoonosis in immunocompromised host.

Zoonotic diseases pose a nosocomial problem, However prompt recognition can successfully protect immunocompromised patients from invasive forms.

This experience emphasizes the problems in recognition of hospital-associated infections.

Control measures must be instituted to limit animal's access to hospital spaces, and prevent the possible fungal contamination of inanimate objects. 
Yassine et al. Tinea corporis caused by Microsporum canis in HIV patient treated for neuromeningeal cryptococcis: report of a nosocomial outbreak

\section{Acknowledgements:}

We appreciate all staff of the department of infectious disease in CHU "Hassani Abdelakader"

\section{Conflict of Interest:}

No conflict of interests is declared.

\section{References}

1. Chekiri-Talbi M, Denning DW. Burden of fungal infections in Algeria. European Journal of Clinical Microbiology;2017:366 .

2. Y SRG. The new species concept in dermatophytes $-\mathrm{A}$ polyphasic approach. Mycopathologia;2008(166):236-256.

3. [3] Odom RB. Common superficial fungal infections in immune-suppressed patient. J Am Acad Dermatol. 1994;p. 569.

4. Primary invasive cutaneaous Microsporum canis in immunocompressed patients. Journal of Clinical Microbiology. 1996;.

5. Bottone EJ. Atlas of the clinical microbiology of infectious diseases: viral, fungal and parasitic agents; 2006.

6. B RMW. Nosocomial Trichophyton tonsurans in a long stay ward. N Z Med J. 1997;p. 277-8.

7. R LMS. The ringworm riddle: an outbreak of Microsporum canis in the nursery. The Pediatric infectous disease. journal. 1993;p. 12-2.

8. J S. Olson-Burgess C, Preuett B, Abdel-Rahman SM. A large outbreak of Trichosporon tonsurans among health care workers in a pediatric hospital. American Journal of infection control;2009(37):43-8.

9. Shah PC, Krajden S, Kane J, Summerbell RC. Tinea corporis caused by Microsporum canis : report of a nosocomial outbreak. European journal of epidemiology 1988;4(1):33-8.

10. M AMM. Nosocomial dermatophytis caused by Microsporum canis in newborn department. Infect Control;1986:7-12.

11. Nosocomial ringworm in neonatal intensive care unit : a nurse and her cat. Infect Control Hosp Epidemiol. 2000;21:605-7. 